\title{
An integrated optimization of quality control chart parameters and preventive maintenance using Markov chain
}

\author{
Farahani, A. ${ }^{a}$, Tohidi, H. ${ }^{\text {b, }}$, Shoja, A. ${ }^{c}$ \\ ${ }^{a}$ Department of Industrial Engineering, Roudehen Branch, Islamic Azad University, Roudehen, Iran \\ ${ }^{b}$ Department of Industrial Engineering, South Tehran Branch, Islamic Azad University, Tehran, Iran \\ 'Department of Mathematics and Statistic, Roudehen Branch, Islamic Azad University, Roudehen, Iran
}

\begin{abstract}
A B S T R A C T
Manufacturing costs are reduced significantly with the integrated optimization of preventive maintenance and quality control. In this paper, a new mixed integer non-linear programming model is presented. This model determines the optimal preventive maintenance interval and the optimal parameters of the $(\bar{X})$ control chart, including the sampling interval and the sample size and the control limit. The production system is considered in the form of a continuous time Markov chain. Formulation of the production process of a machine in the form of a continuous time Markov chain is a breakthrough in the integrated modeling of repair and quality. The goal is to reduce costs per unit time. It is assumed that preventive maintenance can be carried out at several levels either perfect or imperfect. The duration of corrective and preventive maintenance is not negligible. Considering the length of time for maintenance, this model is closer to the real production environment. A numerical example is used to illustrate this new model. Sensitivity analysis was performed to determine the effect of the model parameters on optimal decisions. This analysis further shows the relationship between preventive maintenance and statistical quality control as well as the performance of the new model.
\end{abstract}

\section{ARTICLE INFO}

Keywords:

Maintenance;

Optimization;

Chart control;

Non-linear model;

Markov chain

*Corresponding author:

H_tohidi@azad.ac.ir

(Tohidi, H.)

Article history:

Received 10 December 2017

Revised 22 November 2018

Accepted 3 December 2018

(C) 2019 CPE, University of Maribor. All rights reserved.

\section{References}

[1] Tambe, P.P., Kulkarni, M.S. (2015). A superimposition based approach for maintenance and quality plan optimization with production schedule, availability, repair time and detection time constraints for a single machine, Journal of Manufacturing Systems, Vol. 37, Part 1, 17-32, doi: 10.1016/j.jmsy.2015.09.009.

[2] Hadidi, L.A., Al-Turki, U.M., Rahim, A. (2011). Integrated models in production planning and scheduling, maintenance and quality: A review, International Journal of Industrial and Systems Engineering, Vol. 10, No. 1, 21-50, doi: 10.1504/IJISE.2012.044042.

[3] Pandey, D., Kulkarni, M.S., Vrat, P. (2010). Joint consideration of production scheduling, maintenance and quality policies: A review and conceptual framework, International Journal of Advanced Operations Management, Vol. 2, No. 1-2, 1-24, doi: 10.1504/IJAOM.2010.034583.

[4] Pandey, D., Kulkarni, M.S., Vrat, P. (2011). A methodology for joint optimization for maintenance planning, process quality and production scheduling, Computers \& Industrial Engineering, Vol. 61, No. 4, 1098-1106, doi: 10.1016/i.cie.2011.06.023.

[5] Pandey, D., Kulkarni, M.S., Vrat, P. (2012). A methodology for simultaneous optimisation of design parameters for the preventive maintenance and quality policy incorporating Taguchi loss function, International Journal of Production Research, Vol. 50, No. 7, 2030-2045, doi: 10.1080/00207543.2011.561882.

[6] Liu, L., Yu, M., Ma, Y., Tu, Y. (2013). Economic and economic-statistical designs of an $\bar{X}$ control chart for two-unit series systems with condition-based maintenance, European Journal of Operational Research, Vol. 226, No. 3, 491-499, doi: 10.1016/j.ejor.2012.11.031. 
[7] Xiang, Y. (2013). Joint optimization of $\bar{X}$ control chart and preventive maintenance policies: A discrete-time Markov chain approach, European Journal of Operational Research, Vol. 229, No. 2, 382-390, doi: 10.1016/ j.ejor.2013.02.041.

[8] Zhang, G., Deng, Y., Zhu, H., Yin, H. (2015). Delayed maintenance policy optimisation based on $\bar{X}$ control chart, International Journal of Production Research, Vol. 53, No. 2, 341-353, doi: 10.1080/00207543.2014.923948.

[9] Yin, H., Zhang, G., Zhu, H., Deng, Y., He, F. (2015). An integrated model of statistical process control and maintenance based on the delayed monitoring, Reliability Engineering \& System Safety, Vol. 133, 323-333, doi: 10.1016/ j.ress.2014.09.020.

[10] Bouslah, B., Gharbi, A., Pellerin, R. (2016). Joint economic design of production, continuous sampling inspection and preventive maintenance of a deteriorating production system, International Journal of Production Economics, Vol. 173, 184-198, doi: 10.1016/j.ijpe.2015.12.016.

[11] Tambe, P.P., Kulkarni, M.S. (2016). Selective maintenance optimization under schedule and quality constraints, International Journal of Quality \& Reliability Management, Vol. 33, No. 7, 1030-1059, doi: 10.1108/IJQRM-102014-0153.

[12] Lu, B., Zhou, X., Li, Y. (2016). Joint modeling of preventive maintenance and quality improvement for deteriorating single-machine manufacturing systems, Computers \& Industrial Engineering, Vol. 91, 188-196, doi: 10.1016/ j.cie.2015.11.019.

[13] Nourelfath, M., Nahas, N., Ben-Daya, M. (2016). Integrated preventive maintenance and production decisions for imperfect processes, Reliability Engineering \& System Safety, Vol. 148, 21-31, doi: 10.1016/i.ress.2015.11.015.

[14] Shrivastava, D., Kulkarni, M.S., Vrat, P. (2016). Integrated design of preventive maintenance and quality control policy parameters with CUSUM chart, The International Journal of Advanced Manufacturing Technology, Vol. 82, No. 9-12, 2101-2112, doi: 10.1007/s00170-015-7502-7.

[15] Zhong, J., Ma, Y., Tu, Y.L. (2016). Integration of SPC and performance maintenance for supply chain system, International Journal of Production Research, Vol. 54, No. 19, 5932-5945, doi: 10.1080/00207543.2016.1189104.

[16] Ardakan, M.A., Hamadani, A.Z., Sima, M., Reihaneh, M. (2016). A hybrid model for economic design of MEWMA control chart under maintenance policies, The International Journal of Advanced Manufacturing Technology, Vol. 83, No. 9-12, 2101-2110, doi: 10.1007/s00170-015-7716-8.

[17] Khrueasom, P., Pongpullponsak, A. (2017). The integrated model of the Kolmogorov-Smirnov distribution-free statistic approach to process control and maintenance, Journal of King Saud University-Science, Vol. 29, No. 2, 182-190, doi: 10.1016/i.jksus.2016.04.005.

[18] Salmasnia, A., Abdzadeh, B., Namdar, M. (2017). A joint design of production run length, maintenance policy and control chart with multiple assignable causes, Journal of Manufacturing Systems, Vol. 42, 44-56, doi: 10.1016/ j.jmsy.2016.11.003.

[19] Zhong, J., Ma, Y. (2017). An integrated model based on statistical process control and maintenance for two-stage dependent processes, Communications in Statistics-Simulation and Computation, Vol. 46, No. 1, 106-126, doi: $10.1080 / 03610918.2014 .957841$.

[20] Beheshti Fakher, H., Nourelfath, M., Gendreau, M. (2017). A cost minimisation model for joint production and maintenance planning under quality constraints, International Journal of Production Research, Vol. 55, No. 8, 2163-2176, doi: 10.1080/00207543.2016.1201605.

[21] Rasay, H., Fallahnezhad, M.S., Mehrjerdi, Y.Z. (2017). An integrated model for economic design of chi-square control chart and maintenance planning, Communications in Statistics - Theory and Methods, Vol. 47, No. 12, 2892-2907, doi: 10.1080/03610926.2017.1343848. 


\title{
Integrirana optimizacija parametrov nadzornega grafikona kakovosti in preventivno vzdrževanje z Markovsko verigo
}

\author{
Farahani, A. ${ }^{a}$, Tohidi, H. ${ }^{\text {b. }}{ }^{*}$, Shoja, A. ${ }^{c}$ \\ ${ }^{a}$ Department of Industrial Engineering, Roudehen Branch, Islamic Azad University, Roudehen, Iran \\ ${ }^{\mathrm{b}}$ Department of Industrial Engineering, South Tehran Branch, Islamic Azad University, Tehran, Iran \\ 'Department of Mathematics and Statistic, Roudehen Branch, Islamic Azad University, Roudehen, Iran
}

\begin{abstract}
POVZETEK
Z integrirano optimizacijo preventivnega vzdrževanja in nadzora kakovosti se stroški proizvodnje znatno zmanjšajo. $\mathrm{V}$ tem prispevku je predstavljen nov mešani celoštevilski model nelinearnega programiranja. Model določa optimalni interval preventivnega vzdrževanja in optimalne parametre $(\bar{X})$ nadzornega grafikona, vključno $\mathrm{z}$ intervalom vzorčenja in velikostjo vzorca ter mejo nadzora. Proizvodni sistem je obravnavan v obliki neprekinjene časovne verige Markov. Formulacija proizvodnega procesa določenega stroja v obliki neprekinjene verige Markov je preboj v integriranem modeliranju popravil in kakovosti. Cilj je zmanjšati stroške na enoto časa. Domneva se, da se lahko preventivno vzdrževanje izvaja na več ravneh, popolno ali nepopolno. Trajanje korektivnega in preventivnega vzdrževanja ni zanemarljivo. Glede na dolžino časa za vzdrževanje je predstavljeni model bližje dejanskemu proizvodnemu okolju. Za ponazoritev modela je uporabljen številčni primer. Za določitev vpliva parametrov modela na optimalne odločitve je bila izvedena analiza občutljivosti. Analiza prikazuje povezavo med preventivnim vzdrževanjem in statističnim nadzorom kakovosti ter uspešnostjo predstavljenega modela.
\end{abstract}

\section{PODATKI O ČLANKU}

Ključne besede:

Vzdrževanje;

Optimizacija;

Nadzorni grafikon;

Nelinearni model;

Markovska veriga;

*Kontaktna oseba:

H_tohidi@azad.ac.ir

(Tohidi, H.)

Zgodovina članka:

Prejet 10. decembra 2017

Popravljen 22. novembra 2018

Sprejet 3. decembra 2018 